\title{
ON THE SUMMATION OF FOURIER SERIES
}

BY R. E. A. C. PALEY, W. C. RANDELS, AND M. F. ROSSKOPF

1. Introduction. Suppose that we have a series

$$
u_{1}+u_{2}+u_{3}+\cdots
$$

and an infinite matrix $\left\{\eta_{n, m}\right\},(0 \leqq n<\infty, 1 \leqq m<\infty)$. We say that the series is summable by the method $\{\eta\}$, if the sum

$$
\sum_{n=0}^{\infty} u_{n} \eta_{n, m}
$$

tends to a limit as $m$ tends to infinity.

The method $\{\eta\}$ is said to be regular if, whenever (1) is convergent, (2) tends to the same limit.

Hille and Tamarkin* call a method of summability $F$ effective, if it sums the Fourier series of an integrable function to the value of the function at every point $x$ where the function has a definite value $f(x)$ and where

$$
\phi(t) \equiv f(x+t)+f(x-t)-2 f(x)=o(1),
$$

as $t \rightarrow 0$. They call a method $L$-effective if it sums the Fourier series of an integrable function to the value of the function at every point of the Lebesgue set of the function; that is, at every point $x$ where

$$
\int_{0}^{h}|\phi(t)| d t=o(h)
$$

The object of this paper is to construct a method of summation which is regular and $F$-effective without being $L$-effective. $\dagger$

2. Outline of the Method. We restrict ourselves to methods of

* E. Hille and J. D. Tamarkin, On the summability of Fourier series, Transactions of this Society, vol. 34 (1932), pp. 757-783.

$\dagger$ This problem was proposed to the late R. E. A. C. Paley by Professors Hille and Tamarkin. Before his death, Paley had worked on the problem, leaving an unfinished and incomplete manuscript. On the basis of his work Randels and Rosskopf have proceeded and have been able to solve the problem. 
summation where $\eta_{n, m}$ is of the form $\eta(n / m)$; so that (2) becomes

$$
\sum_{n=0}^{\infty} u_{n} \eta(n / m)
$$

The function $\eta$ need only be defined for rational values of the argument, but it is simpler to suppose that it is defined for all values of $x$ from zero to infinity. We shall suppose that (a) $\eta(x)$ is of bounded variation over $(0, \infty)$, and that $\eta(0)=\eta(+0)=1$. It can easily be shown that the conditions (a) imply the regularity of the method of summation $\{\eta\}$.

Suppose that $\left(b_{1}\right) \quad \eta(x) \rightarrow 0$ as $x \rightarrow \infty$. Then the cosine Fourier transform

$$
K(t)=\left(\frac{2}{\pi}\right)^{1 / 2} \int_{0}^{\infty} \eta(x) \cos t x d x
$$

must exist, and the integral (4) must converge as an improper integral. Suppose further that $\left(b_{2}\right)$

$$
\int_{0}^{\infty}|K(t)| d t<\infty \text {. }
$$

Hille and Tamarkin* have shown that the existence of the integral (5) is necessary and sufficient in order that a method of summation which satisfies (a) and $\left(b_{1}\right)$ be $F$-effective.

The outline of our method is as follows. We construct a kernel $K(t)$ which furnishes a method of summability which is $F$ effective. Then we construct a particular function $f(x), f(0)=0$, the point $x=0$ belonging to the Lebesgue set of $f(x)$, such that the method $\{\eta\}$ does not sum the Fourier series of $f(x)$ to zero at $x=0$.

3. Construction of the Kernel. The kernel which we shall use is defined by

$$
K(t)=\sum_{n=1}^{\infty} K_{n}(t)
$$

* In an unpublished paper presented to the Society; see E. Hille and J. D. Tamarkin, On the summability of Fourier series, IV, this Bulletin, vol. 39, Abstract 181, May (1933). 
where

$$
K_{n}(t)= \begin{cases}+\frac{1}{t n^{3 / 2}}\left(t-2^{n}+n^{1 / 2}\right), & 2^{n}-n^{1 / 2} \leqq t \leqq 2^{n} \\ -\frac{1}{t n^{3 / 2}}\left(t-2^{n}-n^{1 / 2}\right), & 2^{n} \leqq t \leqq 2^{n}+n^{1 / 2} \\ 0, & \text { elsewhere },\end{cases}
$$

for $n=1,2, \cdots$. Then it can easily be proved that

$$
K(t) \subset L, \quad K^{*}(t) \equiv t K(t) \subset L_{2},
$$

and that $K(t)$ is of bounded variation over $(0, \infty)$. We define $A$ by

$$
A=\left(\left(\frac{2}{\pi}\right)^{1 / 2} \int_{0}^{\infty} K(t) d t\right)^{-1} .
$$

Let us define $\eta(x)$ as

$$
\eta(x)=A\left(\frac{2}{\pi}\right)^{1 / 2} \int_{0}^{\infty} K(t) \cos t x d t ;
$$

then $\eta(0)=1$. Now we consider the Fourier series of an even, integrable, periodic function $f(x)$,

$$
f(x) \sim \frac{a_{0}}{2}+\sum_{n=1}^{\infty} a_{n} \cos n x
$$

then if we replace $x$ by $t / m$, multiply the Fourier series of $f(t / m)$ by $A(2 / \pi)^{1 / 2} K(t)$, and integrate from zero to infinity, we obtain

$$
\begin{gathered}
A\left(\frac{2}{\pi}\right)^{1 / 2} \int_{0}^{\infty} K(t) f(t / m) d t=A \frac{a_{0}}{2}\left(\frac{2}{\pi}\right)^{1 / 2} \int_{0}^{\infty} K(t) d t \\
+A\left(\frac{2}{\pi}\right)^{1 / 2} \int_{0}^{\infty} K(t)\left(\sum_{n=1}^{\infty} a_{n} \cos \frac{n}{m} t\right) d t \\
=\frac{a_{0}}{2}+\sum_{n=1}^{\infty} a_{n} \eta(n / m)
\end{gathered}
$$


This step is legitimate since $K(t)$ is of bounded variation over $(0, \infty) \cdot \dagger$

By Plancherel's theorem for Fourier integrals, since $K^{*}(t)$ $\subset L_{2}$, we have

$$
\eta_{0} *(x) \sim A\left(\frac{2}{\pi}\right)^{1 / 2} \int_{0}^{\infty} K^{*}(t) \sin x t d t \subset L_{2}
$$

over $(0, \infty)$. Furthermore if we denote by $\eta_{n}{ }^{*}(x)$ the Fourier transform of $K_{n}^{*}(x)$, we can write

$$
\begin{aligned}
\eta^{*}(x)=\sum_{n=1}^{\infty} \eta_{n}^{*}(x) & =A \sum_{n=1}^{\infty} \int_{2^{n}-n^{1 / 2}}^{2^{n}+n^{1 / 2}} K_{n}^{*}(t) \sin x t d t \\
& =A \sum_{n=1}^{\infty} \frac{2 \sin 2^{n} x\left(1-\cos n^{1 / 2} x\right)}{x^{2} n^{3 / 2}} .
\end{aligned}
$$

It is easily seen that $\eta^{*}(x)$ exists everywhere; hence $\eta_{0}^{*}(x)$ $=\eta^{*}(x)$ almost everywhere. Now clearly $\eta^{*}(x)$ is in tegrable over $(a, \infty), a>0$, and $\eta_{0}{ }^{*}(x)$ is integrable over the interval $(0, a)$; hence $\eta^{*}(x) \subset L$ over the interval $(0, \infty)$. We write

$$
\int_{0}^{x} \eta^{*}(u) d u=\lim _{\delta \rightarrow 0} \int_{\delta}^{x}\left\{\sum_{n=1}^{\infty} \eta_{n}^{*}(u)\right\} d u .
$$

The sum (7) is dominated by

$$
A \sum_{n=1}^{\infty} \frac{1}{x^{2} n^{3 / 2}}
$$

which belongs to $L$ over $(\delta, x)$; hence we may integrate (8) termwise; this gives

$$
\int_{0}^{x} \eta^{*}(u) d u=\lim _{\delta \rightarrow 0} \sum_{n=1}^{\infty} A \int_{\delta}^{x} d u \int_{0}^{\infty} K_{n}^{*}(t) \sin u t d t .
$$

Since $K_{n}{ }^{*}(t)$ vanishes except on a finite interval, we may interchange the order of integration and obtain

† See E. V. Hobson, The Theory of Functions of a Real Variable and the Theory of Fourier's Series, 2d edition, 1926, vol. II, p. 583. Also G. H. Hardy, Notes on some points in the integral calculus, LV, Messenger of Mathematics, vol. 51 (1922), pp. 186-192. 


$$
\begin{aligned}
\int_{0}^{x} \eta^{*}(u) d u & =\lim _{\delta \rightarrow 0} \sum_{n=1}^{\infty} A \int_{0}^{\infty} K_{n}^{*}(t) \frac{\cos \delta t-\cos x t}{t} d t \\
& =\lim _{\delta \rightarrow 0} \sum_{n=1}^{\infty} A \int_{0}^{\infty} K_{n}(t)(\cos \delta t-\cos x t) d t \\
& =\lim _{\delta \rightarrow 0} A \int_{0}^{\infty} K(t)(\cos \delta t-\cos x t) d t \\
& =A \int_{0}^{\infty} K(t) d t-\eta(x),
\end{aligned}
$$

since $K(t)$ is integrable. Consequently the right-hand member of (9), as an indefinite integral of an integrable function, is of bounded variation; hence $\eta(x)$ must be of bounded variation. Furthermore, $\eta(x) \rightarrow 0$ as $x \rightarrow \infty$, since it is the Fourier transform of an absolutely integrable function.

Therefore the method of summability defined by $\eta(x)$ is regular and $F$-effective.

4. Construction of the Function. However, this method is not $L$-effective. For consider the even periodic function

$$
f(x)=\left\{\begin{array}{l}
2^{n}, 2^{-n}-\frac{4^{-n}}{2 \log (n+1)} \leqq x \leqq 2^{-n}+\frac{4^{-n}}{2 \log (n+1)}, \\
0, \text { elsewhere in }(0,2 \pi),
\end{array}\right.
$$

so that $f(0)=0$.

Let us take for consideration the point $x=0$. This point belongs to the Lebesgue set of $f(x)$ since

$$
\begin{aligned}
\int_{0}^{t} f(x) d x & =O\left(\sum_{n=\left[-\log _{2} t\right]-1}^{\infty} \frac{2^{-n}}{\log (n+1)}\right) \\
& =O\left(\frac{2^{-\left[-\log _{2} t\right]+2}}{\log \left(\left[-\log _{2} t\right]\right)}\right) \\
& =O\left(\frac{t}{\log \left(-\log _{2} t\right)}\right)=o(t)
\end{aligned}
$$

as $t \rightarrow 0$. At this point $x=0$, then, the limit of (6) would have to be zero if the method were $L$-effective.

Consider the integral (6), 


$$
A\left(\frac{2}{\pi}\right)^{1 / 2} \int_{0}^{\infty} K(t) f(t / m) d t>A\left(\frac{2}{\pi}\right)^{1 / 2} \int_{0}^{m} K(t) f(t / m) d t .
$$

Replace $m$ by $2^{m}$; then our function $f(x)$ becomes

$$
f\left(t / 2^{m}\right)=\left\{\begin{array}{lr}
2^{n}, 2^{m-n}-\frac{2^{m} 4^{-n}}{2 \log (n+1)} \leqq t \leqq 2^{m-n}+\frac{2^{m} 4^{-n}}{2 \log (n+1)}, \\
0, \text { elsewhere in }(0,2 \pi), & (n=1,2,3, \cdots) .
\end{array}\right.
$$

Hence

$$
A\left(\frac{2}{\pi}\right)^{1 / 2} \int_{0}^{2^{m}} K(t) f\left(t / 2^{m}\right) d t>A\left(\frac{2}{\pi}\right)^{1 / 2} \sum_{n=1}^{[m / 2]} \int_{J_{n}} K(t) f\left(t / 2^{m}\right) d t,
$$

where

$$
J_{n} \equiv\left(2^{n}-\frac{2^{m} 4^{n-m}}{2 \log (m-n+1)}, 2^{n}+\frac{2^{m} 4^{n-m}}{2 \log (m-n+1)}\right) .
$$

But over $J_{n}(n \leqq m / 2), K(t)>(1 / 4)\left(2^{-n} / n\right)$; therefore we have

$$
\begin{aligned}
A\left(\frac{2}{\pi}\right)^{1 / 2} \sum_{n=1}^{[m / 2]} \int_{J_{n}} K(t) f\left(t / 2^{m}\right) d t \\
>A\left(\frac{2}{\pi}\right)^{1 / 2} \frac{1}{4} \sum_{n=1}^{[m / 2]} \frac{2^{-n}}{n} \frac{2^{m} 4^{n-m}}{\log (m-n+1)} 2^{m-n} \\
=A\left(\frac{2}{\pi}\right)^{1 / 2} \frac{1}{4} \sum_{n=1}^{[m / 2]} \frac{1}{n \log (m-n+1)} \\
>A\left(\frac{2}{\pi}\right)^{1 / 2} \frac{1}{4 \log m} \sum_{n=1}^{[m / 2]} \frac{1}{n} \sim A\left(\frac{2}{\pi}\right)^{1 / 2} \frac{1}{4},
\end{aligned}
$$

as $m \rightarrow \infty$.

Hence the method $\{\eta\}$ is not $L$-effective.

BROWN UNIVERSITY 